\title{
Exploring Factors Affecting Behavioral, Cognitive and Emotional Engagement of International Undergraduate Students in China 来华本科留学生学习性投入的影响因素研究 基于行为、认知和情感三维度
}

\author{
Meiqiong Gong 烡美琼 \\ Shanghai Jiao Tong University, China \\ 上海交通大学，中国 \\ Yuhao Cen 岑逾豪 \\ Shanghai Jiao Tong University, China \\ 上海交通大学，中国
}

\begin{abstract}
Student engagement is one of the critical measures assessing the quality of international student education. This survey study examined the behavioral, cognitive and emotional engagement of international students at a research-intensive university on the east coast of China. The findings showed the interrelationship of the behavioral, cognitive and emotional dimensions of student engagement. The research revealed that individual characteristics had significant effects on emotional engagement, while having no effect on behavioral or cognitive engagement. Male students, first-generation students, and students enrolling in degree programs displayed a lower level of emotional engagement compared with their counterparts. The research also found that supportive campus environment and frequent student-faculty interactions were positively related to the three dimensions of student engagement, whereas peer interactions had no effect on any of the dimensions.
\end{abstract}

摘要：来华留学生的学习性投入水平，是衡量高校留学生教育质量的重要方式。 本文将学习性投入划分为行为投入、高阶认知投入、情感投入三维度, 基于中国 东海岸一所研究型大学来华本科留学生的调查数据, 对留学生的学习性投入水平 与影响因素进行描述性统计与回归分析。研究发现, 留学生三维度的学习性投入 显著正相关。个人背景特征对留学生的情感投入存在显著影响, 但对行为和高阶 认知投入没有影响。男性、第一代大学生、攻读学位项目的留学生均呈现较低的 
情感投入。校园环境支持度和师生互动因素对学习性投入三维度都具有显著影响, 但生生互动对学习性投入各维度没有影响。

Keywords: international students in China, student engagement, behavioral engagement, cognitive engagement, emotional engagement 关键词: 来华留学生, 学习性投入, 行为投入, 高阶认知投入, 情感投入

\section{引言}

在中国建设世界一流大学、增强国际竞争力的目标背景下, 来华留学生的数量不 断攀升。2010 年, 教育部颁布的《留学中国计划》提出了 “扩大规模, 优化结 构, 规范管理, 保证质量” 的来华留学生工作指导方针, 表示到 2020 年, 接受 高等学历教育的留学生要达到 15 万人。2007-2017 年间, 高校来华留学生人数逐 年增加, 年平均增长率为 $11.6 \%$ (图 1)。高校在通过国家政策支持扩大来华留 学生规模的同时, 也应通过内涵建设提升来华留学生教育的质量, 使来华留学生 在中国学有所获、学有所成, 这也恰是高校学生群体一一包括来华留学生一一所 关心的学习成果和求学过程的质量保障。

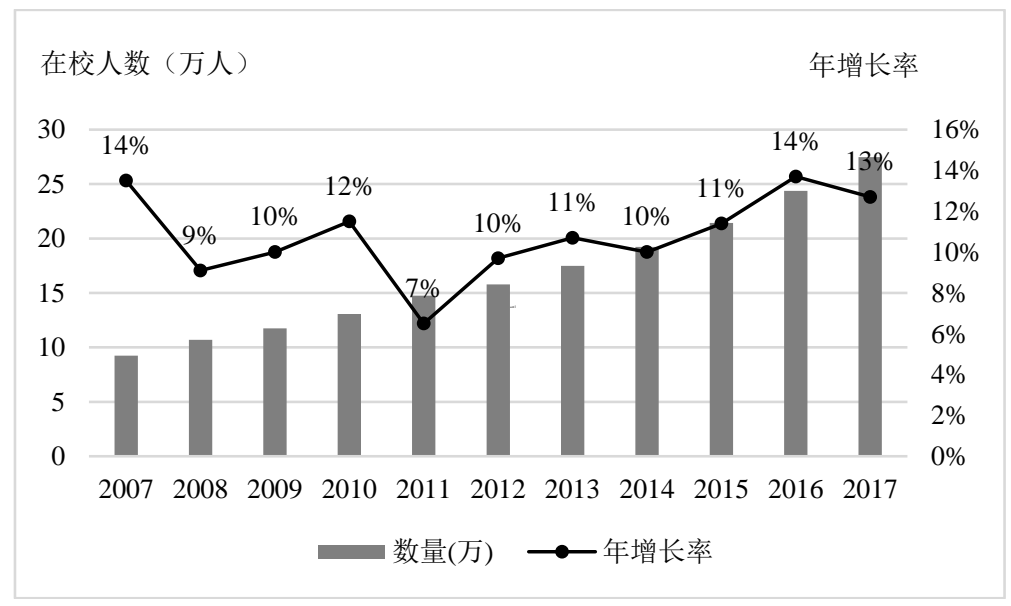

图 1.2007-2017 年中国高校来华留学生在校人数及年增长率

数据来源：中华人民共和国教育部, 2007-2017.

高等教育质量评估范式已经发生改变, 从注重学校的资源投入转变为关注学 生的投入与产出 (Coates, 2005; 孙超, 2009) 。学生学习成果产出的评价不易, 评 价学习性投入 (student engagement) 成为衡量高等教育质量的重要手段。正如 Kuh (2001) 等学者所指出的, 提高教育质量的核心要素是提升学生的学习投入 度。学习性投入是衡量教育质量与学生发展的重要内容, 提高学习性投入水平是 提升教育质量最有效的方式之一。

基于上述认识, 本研究以来华留学生为研究对象, 围绕以下问题展开相应研 究: (1) 来华留学生的学习性投入水平如何, 包括在行为、认知、情感三维度 
的学习性投入水平分别如何? (2) 来华留学生的学习性投入三维度, 行为投入、 高阶认知投入、情感投入, 分别受哪些个体和院校因素影响?

\section{文献评述}

学界对于学习性投入的概念尚未形成定论, 但其内涵演变经历了从单维到多维的 发展过程。学习性投入最初起源于 Tyler 提出的 “任务投入时间” （time on task） 和 Pace 提出的 “努力质量” （quality of effort），Astin 在 20 世纪 80 年代提出的 “学生参与”（student involvement）促进了学习性投入概念的进一步发展 （Axelson \& Flick, 2010）。在 20 世纪 90 年代, 有人认为学习性投入是学生的心 理投入（Newmann, 1991），也有学者认为它包括行为投入和情感投入两种形式

（Finn, 1993）。Kuh（2001）则从学校与个体互动和建构的角度对学习性投入进 行定义, 认为它既包括学生自主投入的时间、精力与努力, 又包括院校为促进学 生学习与发展而投入的资源与努力。Fredricks 等人（2004）将学习性投入划分为 行为投入、认知投入、情感投入三个维度, 这一观点现已成为学界的主流观点。 岑逾豪 $(2014,2016)$ 通过对中国大学生的实证研究发现, 情感投入是学习性投 入的重要组成部分。

已有研究表明, 影响学生学习性投入的因素主要包括院校和学生两个层面。 院校层面因素指学校为学生提供的环境设施、教学资源、人际互动等, 学生层面 的因素包括学生的背景特征等。院校层面的影响因素包括教师相关因素、生生互 动、学校环境与政策等方面。教师在提升学生投入方面扮演着重要角色, 教师的 教学策略、与学生的互动、给学生提供的任务特征、强调高阶认知活动等方面都 会影响到学生的学习性投入水平（Umbach \& Wawrzynski, 2005; Zepke, 2011）。 同伴关系对学生的学业投入和联课活动（如本科生科研、竞赛等）的投入也会产 生影响。良好的同伴关系对学习性投入产生积极影响; 当学生被边缘化、被同伴 排挤和欺凌时, 学习性投入显著降低（Juvonen, Espinoza, \& Knifsend, 2012）。此 外, 校园环境、学校的政策和举措、整体氛围对学生投入也会产生影响。学校的 政策调整、提供支持与服务的程度等都会影响学生的学习性投入水平 (Pike et al., 2006; Porter, 2006; Schuetz, 2008）。

个体层面的因素包括学生的个人背景、就读经验特征等方面（Bauer \& Liang, 2003; Brint et al., 2008; Hu \& Kuh, 2002; Kuh, Hu \& Vesper, 2000; Pike, 2000; 靳敏 \& 胡寿平, 2018）。中外研究均发现学习性投入水平存在显著的性别和年级差异, 女生的学习投入水平显著高于男生（Kuh et al., 2006; 靳敏 \& 胡寿平, 2018; 张信勇, 市小华, \& 徐光兴, 2008），高年级学生的投入水平高于低年级学生（Astin, 1977; Pike, Schroeder, \& Berry, 1997; 吴素梅 \& 宋彩萍, 2010）。学生的家庭背景、家庭 的社会经济地位也会影响学生的学习性投入水平, 但众多研究的结论并未达成一 致（Pike \& Kuh, 2005; Miliszewska \& Horwood, 2004）。学生的学习动机、自我 效能感等与学习性投入水平正相关（D. Fazey \& J. Fazey, 2001; Schuetz, 2008）。

上述研究多以本国高校学生为研究对象, 针对来华留学生的学习性投入研究 较少。此外, 在高等教育领域, 鲜有将情感维度纳入学习性投入并进行系统测量 的研究。因此, 本文试图突破上述局限, 以来华留学生为研究对象, 通过问卷调 查和定量分析, 探索其在行为、认知、情感三维度的水平及影响因素。 


\section{研究设计}

\section{概念操作化}

本研究将学习性投入划分为行为、高阶认知、情感三维度, 结合各学者的观 点, 将上述三维度定义为:

1. 行为投入: 学生对学业学习及学业相关活动所投入的时间;

2. 高阶认知投入: 学生在参与学习或学业相关活动中采用高阶思维策略, 包括使用理解、分析、综合、判断、运用等策略完成任务 (Krathwohl \& Anderson, 2009; Bloom,1956; 岑逾豪, 2014);

3. 情感投入: 指个体在学习时所具有的充沛精力和良好的心理韧性, 进 一步细分为活力 (vigor)、投身 (dedication)、专注 (absorption) 三维 度。其中, 活力表现为在学习时精力充沛, 愿意付出努力, 在学习上 遇到困难也坚持不解; 投身则是指全身心投入到学习中的状态, 并在 过程中体验到意义与价值、热情、自豪与挑战; 专注表现为学习者全 神贯注地投入到学习中, 感觉时间过得很快并很难从这种状态中抽离 出来（Schaufeli et al., 2002; Schaufeli, Bakker, \& Salanova, 2006)。

\section{研究工具}

本研究采用中英双语版问卷, 使用已发表的成熟量表测量学习性投入的三个 维度 (表 1)。

表 1. 研究工具构成及信度

\begin{tabular}{|c|c|c|c|c|}
\hline 子量表 & 组成部分 & 题项示例 & 赋值定义 & 信度（ $\alpha)$ \\
\hline $\begin{array}{c}\text { 行为投入 } \\
\text { (3 题) }\end{array}$ & $\begin{array}{c}\text { 学业学习 } \\
\text { 中文语言学习 } \\
\text { 学业相关活动 }\end{array}$ & $\begin{array}{l}\text { 一周花在中文 } \\
\text { 语言学习的时 } \\
\text { 间 }\end{array}$ & $\begin{array}{l}1=0 \text { 小时/周; } \\
8=>30 \text { 小时/周 }\end{array}$ & -- \\
\hline $\begin{array}{c}\text { 高阶认知投入 } \\
\text { (5 题 })\end{array}$ & $\begin{array}{l}\text { 理解 } \\
\text { 分析 } \\
\text { 综合 } \\
\text { 判断 } \\
\text { 运用 }\end{array}$ & $\begin{array}{l}\text { 理解学科领域 } \\
\text { 的事实、概念 } \\
\text { 和观点 }\end{array}$ & $\begin{array}{c}1=\text { 从未; } \\
4=大 \text { 多数时候 }\end{array}$ & 0.80 \\
\hline $\begin{array}{c}\text { 情感投入 } \\
\text { （9题） }\end{array}$ & $\begin{array}{c}\text { 活力维度 } \\
\text { (3 题) } \\
\text { 投身维度 } \\
\quad(3 \text { 题 }) \\
\text { 专注维度 } \\
\text { (3 题) }\end{array}$ & $\begin{array}{l}\text { 学习时, 我感 } \\
\text { 到自己迸发出 } \\
\text { 能量 } \\
\text { 学习使我振奋 } \\
\text { 我沉浸于我的 } \\
\text { 学习中 }\end{array}$ & $\begin{array}{l}1=\text { 从不; } \\
7=\text { 总是 }\end{array}$ & $\begin{array}{l}0.86 \\
0.78\end{array}$ \\
\hline
\end{tabular}

其中，行为投入和高阶认知投入参考美国 “全国大学生学习投入调查” （NSSE）的子量表; 情感投入采用乌特勒支工作投入一学生版（Utrecht Work 
Engagement Scale-Student）量表。中文翻译参考了经信效度检验的汉化版量表 （岑逾豪, 2014; 张轶文 \& 甘怡群, 2005; 方来坛, 时勘, \& 张风华, 2008）。正式施 测前, 研究者通过访谈进一步调整和完善问卷。数据收集完毕后对子量表进行信 度检验，各子量表信度良好（表 1）。

\section{数据来源}

本研究在上海一所综合性研究型大学 ( $\mathrm{S}$ 校) 展开。 $\mathrm{S}$ 校是 985 工程和 “双 一流” 建设高校, 其留学生的来源国超过 100 个国家, 本科留学生总数约 1000 人。2019 年 4-5 月, 我们在该校两个校区留学生集中的教学及居住区发放问卷, 多数留学生选择扫二维码填写网络问卷, 少数留学生填写了纸质问卷。共发放问 卷 350 份, 回收有效问卷 202 份。调查样本来自 47 个国家; 平均年龄 21 岁; 男 生占 $62.4 \%$; 理科生占 $41.1 \%$; 攻读学位留学生占 $85.1 \%$; 大一学生占 $(52.5 \%)$, 比例最高, 未划分年级的学生大多为非学位项目留学生（表 2）。

\section{表 2. 调查样本特征描述}

\begin{tabular}{|c|c|c|c|c|c|c|c|}
\hline \multicolumn{2}{|c|}{ 变量类别 } & $\mathrm{N}$ & $\%$ & \multicolumn{2}{|c|}{ 变量类别 } & $\mathrm{N}$ & $\%$ \\
\hline \multirow{2}{*}{ 性别 } & 男 & 126 & 62.4 & \multirow{2}{*}{$\begin{array}{l}\text { 项目 } \\
\text { 类型 }\end{array}$} & \multirow{2}{*}{$\begin{array}{l}\text { 学位项目 } \\
\text { 非学位项目 }\end{array}$} & 172 & 85.1 \\
\hline & 女 & 76 & 37.6 & & & 30 & 14.9 \\
\hline \multirow{5}{*}{ 年级 } & 大一 & 106 & 52.5 & \multirow{9}{*}{$\begin{array}{l}\text { 来自 } \\
\text { 地区 }\end{array}$} & 东亚 & 38 & 18.8 \\
\hline & 大二 & 29 & 14.3 & & 东南亚 & 79 & 39.1 \\
\hline & 大三 & 28 & 13.9 & & 南亚 & 6 & 3.0 \\
\hline & 大四 & 29 & 14.3 & & 中西亚 & 14 & 6.9 \\
\hline & 未划分 & 10 & 5.0 & & 欧洲 & 37 & 18.3 \\
\hline \multirow{2}{*}{ 学科 } & 理工科 & 85 & 42.1 & & \multirow{4}{*}{$\begin{array}{l}\text { 美洲 } \\
\text { 澳洲 } \\
\text { 非洲 }\end{array}$} & 18 & 12.9 \\
\hline & 非理工科 & 117 & 57.9 & & & 8 & 4.0 \\
\hline 第一代 & 是 & 87 & 43.1 & & & 2 & 1.0 \\
\hline 大学生 & 否 & 115 & 56.9 & & & & \\
\hline
\end{tabular}

\section{研究发现}

本研究通过对样本进行描述和推断统计分析, 分析来华留学生在学习性投入及其 三个维度上的水平，并对其影响因素进行分析。

\section{来华留学生学习性投入水平分析}

就行为投入维度而言, 超过两成留学生每周在学业学习上的投入大于 30 小 时, $2 \%$ 的留学生每周在学业学习上的投入时间为 0 小时。相比于中文学习、联 课活动（如本科生科研、竞赛等）的投入水平，留学生在课业学习上的时间投入 相对较高。 


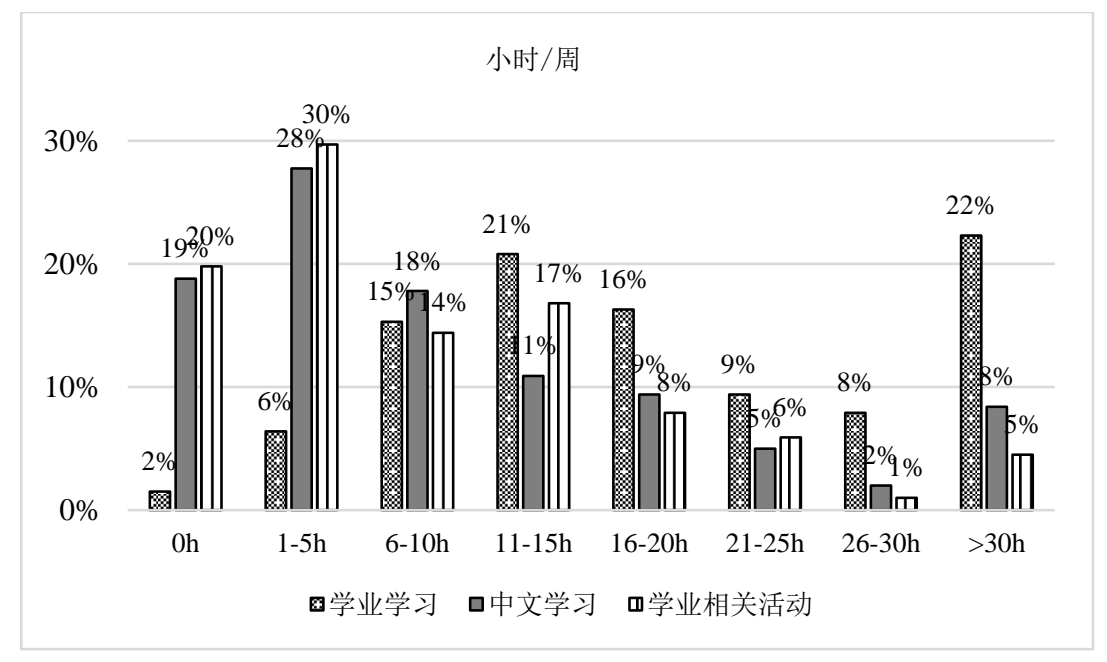

图 2. 来华留学生行为投入水平分布

就认知投入维度而言, 来华留学生使用不同认知策略的水平存在差异, 采用 “理解” 策略最多（3.15），使用 “运用” 策略最少（2.75）（表 3）。

表 3. 来华留学生的高阶认知投入水平

\begin{tabular}{ccc}
\hline 高阶认知投入 (最低值 1, 最高值 4) & 平均值 & 标准差 \\
\hline 理解 & 3.15 & 0.77 \\
分析 & 2.99 & 0.58 \\
综合 & 2.82 & 0.63 \\
判断 & 2.80 & 0.74 \\
运用 & 2.75 & 0.61 \\
\hline
\end{tabular}

就情感投入维度而言, 本科留学生更倾向于赞同 “学习使我振奋” 等投身维 度的陈述（4.28），表达的 “沉浸于我的学习” 等专注水平最低（2.91）（表 4)。

表 4. 来华留学生的情感投入水平

\begin{tabular}{ccc}
\hline 情感投入 (最低值 1, 最高值 7) & 平均值 & 标准差 \\
\hline 活力 & 3.88 & 1.35 \\
投身 & 4.28 & 1.42 \\
专注 & 2.91 & 0.54 \\
\hline
\end{tabular}

表 5 描述了不同生源地留学生的学习性投入水平。除样本数过少的非洲学生 (仅 2 人) 外, 中西亚留学生投入学习的时间最长 (4.36), 澳洲学生投入最少 （3.13）；南亚留学生的情感投入最高（4.87），东亚地区留学生情感投入最低 （3.62）。单因素方差分析结果显示, 不同来源地留学生在三类学习性投入水平 上均无显著差别。 
表 5. 不同地区留学生的学习性投入水平

\begin{tabular}{lccc}
\hline \multicolumn{1}{c}{ 地区 (人数) } & 行为投入 & 高阶认知投入 & 情感投入 \\
\hline 东亚 $(38$ 人) & 3.74 & 2.86 & $\mathbf{3 . 6 2}$ \\
东南亚 $(79$ 人) & 3.71 & 2.94 & 3.87 \\
南亚 $(6$ 人) & 3.56 & $\mathbf{3 . 0 7}$ & $\mathbf{4 . 8 7}$ \\
中西亚 $(14$ 人) & 4.36 & 2.76 & 4.67 \\
欧洲 $(37$ 人) & 4.07 & 2.89 & 4.26 \\
美洲 $(18$ 人) & 3.94 & 3.02 & 3.88 \\
澳洲 $(8$ 人) & $\mathbf{3 . 1 3}$ & 2.78 & 3.67 \\
非洲 $(2$ 人) & $\mathbf{5 . 6 7}$ & $\mathbf{2 . 4 0}$ & 4.00 \\
\hline
\end{tabular}

\section{学习性投入各维度间的关系}

对学习性投入的三个维度进行相关分析发现, 两两之间呈现显著相关（表 6）。其中高阶认知投入与情感投入之间的相关性最强，行为投入与情感投入之 间的相关性最弱。学习过程中, “身”、“心”、“脑” 任何一个方面的投入水 平提升, 可能带动其他两个方面的投入。

表 6. 学习性投入三维度之间的相关性分析

\begin{tabular}{llcc}
\hline 学习性投入维度 & 1 & 2 & 3 \\
\hline 1.行为投入 & 1 & $0.300^{* *}$ & $0.275^{* *}$ \\
2. 高阶认知投入 & & 1 & $0.394^{* *}$ \\
3.情感投入 & & & 1 \\
\hline
\end{tabular}
** $P<0.01$ 。

\section{学习性投入各维度的影响因素分析}

本研究应用多元线性回归模型来分析学习性投入各维度的影响因素。以来华 本科留学生的行为投入、高阶认知投入和情感投入为因变量, 以学生背景信息 （表 2) 和院校层面的变量 (表 7) 为自变量进行回归分析。

为保证多元线性回归模型的科学性, 对自变量是否存在多重共线性进行了检 测。一般来说, 容忍度小于 0.1 或者方差膨胀因子 (VIF) 大于 10 时, 自变量间 可能存在多重共线性问题。构建的三个模型中，容忍度均大于 0.1 且 VIF 小于 10 , 说明自变量间不存在多重共线性问题（吴明隆, 2010）。 
表 7. 院校层面变量

\begin{tabular}{clc}
\hline \multicolumn{1}{c}{ 变量名 } & \multicolumn{1}{c}{ 题项示例 } & \multicolumn{1}{c}{ 赋值定义 } \\
\hline \multirow{2}{*}{ 校园环境支持度 (4 题) } & 学校为你的学业成功 & $1=$ 不强调; \\
& 提供支持帮助 & $4=$ 非常强调 \\
师生互动 (6 题) & 和专业教师讨论成绩 & $1=$ 从不; \\
& 评分或课业 & $4=$ 十分频繁 \\
& 与其他同学建立起了 & $1=$ 非常不同意; \\
生生互动 (6 题) & 密切的人际关系 & $5=$ 非常同意 \\
\hline
\end{tabular}

由表 8 可见, 影响来华留学生学习性行为投入的因素包括校园环境支持度、 师生互动因素。校园环境支持度、师生互动对于留学生的学习行为投入具有显著 的正向影响。同样的, 校园环境支持度和师生互动这两项环境因素对留学生的高 阶认知投入水平也具有显著的正向影响。

表 8. 学习性投入影响因素的回归分析结果

\begin{tabular}{|c|c|c|c|c|c|c|}
\hline \multirow{3}{*}{ 自变量 } & \multicolumn{6}{|c|}{ 因变量 } \\
\hline & \multicolumn{2}{|c|}{ 行为投入 } & \multicolumn{2}{|c|}{ 高阶认知投入 } & \multicolumn{2}{|c|}{ 情感投入 } \\
\hline & Coeff. & SE Sig. & Coeff. & SE Sig. & Coeff. & SE Sig. \\
\hline 常量 & & 0.252 & & 0.000 & & 0.421 \\
\hline 性别(女性) ${ }^{\mathrm{a}}$ & 0.137 & 0.053 & 0.090 & 0.201 & 0.155 & 0.018* \\
\hline 年龄 ～～～～ & 0.034 & 0.629 & -0.091 & 0.205 & 0.038 & 0.566 \\
\hline 第一代大学生 b & -0.001 & 0.982 & -0.059 & 0.366 & -0.132 & $0.028 *$ \\
\hline 项目类型(学位项目) ${ }^{\mathrm{c}}$ & -0.030 & 0.672 & 0.047 & 0.507 & -0.139 & $0.033 *$ \\
\hline 学科（理工类）d & -0.034 & 0.636 & -0.072 & 0.326 & -0.095 & 0.157 \\
\hline 成绩排名前 $25 \%$ e & 0.114 & 0.189 & 0.151 & 0.083 & 0.139 & 0.084 \\
\hline 成绩排名 $25 \%-50 \% \mathrm{e}$ & 0.038 & 0.645 & 0.050 & 0.546 & 0.100 & 0.188 \\
\hline 成绩排名 $50 \%-75 \% \mathrm{e}$ & 0.074 & 0.378 & -0.014 & 0.866 & 0.020 & 0.799 \\
\hline 成绩排名不清楚 e & 0.065 & 0.479 & -0.039 & 0.677 & 0.139 & 0.103 \\
\hline 校园环境支持度 & 0.235 & $0.002 * *$ & 0.204 & $0.007 * *$ & 0.233 & $0.001 * *$ \\
\hline 师生互动 ～～ & 0.295 & $0.000 * *$ & 0.244 & $0.001 * *$ & 0.381 & $0.000 * *$ \\
\hline 生生互动 & -0.086 & 0.208 & 0.053 & 0.439 & 0.079 & 0.210 \\
\hline$F$ & \multicolumn{2}{|c|}{4.768} & \multicolumn{2}{|c|}{4.562} & \multicolumn{2}{|c|}{8.306} \\
\hline 调整后的 $R^{2}$ & \multicolumn{2}{|c|}{0.184} & \multicolumn{2}{|c|}{0.175} & \multicolumn{2}{|c|}{0.304} \\
\hline
\end{tabular}

注: a参照组: 男性; b参照组: 非第一代大学生; ${ }^{c}$ 参照组: 非学位项目; d参照 组: 非理工类; $\mathrm{e}$ 参照组: 成绩排名后 $25 \%$ 。因单因素方差分析表明不同生源国 的留学生投入并无差异, 地区变量未纳入回归分析。

$* P<0.05, * * P<0.01$ 。 
由表 8 可见, 影响情感投入的因素更为多元, 除了院校环境中的校园环境支 持度和师生互动, 还包括学生个体背景特征, 如性别、是否第一代大学生、是否 攻读学位等。来华留学生的情感投入具有显著的性别差异, 女生的情感投入显著 高于男生。家庭背景对留学生的情感投入具有显著影响：第一代大学生的情感投 入水平显著低于非第一代大学生。攻读学位留学生的情感投入要显著低于攻读非 学位项目的留学生。

\section{讨论与启示}

面向中国国内学生的高等教育历经了从规模扩大到质量提升的转变, 但目前大部 分高校的来华留学生教育的重心仍停留在 “扩大规模、优化结构” 阶段。本研究 通过探索来华留学生的学习性投入水平及其影响因素, 希望为中国高校留学生教 育围绕 “以学生的学习与发展为中心” 并走向内涵发展提供一些启示。

来华留学生学习性投入的行为、认知和情感维度相互关联与影响。行为投入 是外在的、显性的学习性投入形式，高阶认知投入和情感投入则是较为内隐的投 入形式。相比于行为投入与高阶认知投入，情感投入是一种更深层次的投入形式， 需要学习者对学习内容和任务抱有认同与热情。强调在学业学习和中文学习中投 入大量时间, 布置具有挑战性的高阶认知学习任务, 能够带动留学生的情感投入。

来华留学生的部分个人背景特征（如性别和父母学历）对情感投入水平具有 显著影响, 但对留学生的学习时间投入、高阶认知投入不存在显著影响。国外研 究发现学生的背景特征通常只能解释学习性投入极小部分的差异 (Pike, 1999; Pike \& Killian, 2001)。这些不可改变的学生个体特质对学习性投入的影响微乎其 微，对高等教育的决策者和实践者而言是利好消息。

此外, 本研究发现来华攻读学位留学生的情感投入低于非学位留学生。非学 位留学生包括短期交流生和学习语言的留学生。一方面, 长期学习的留学生度过 来到中国的 “蜜月期” ，新鲜感和刺激感褪去后，对学习的热情可能回落。另一 方面, 中国高校的汉语学习和短期交流项目有一定建设历史, 而面向留学生的学 位项目起步较晚, 相比前者提供的教育资源尚不完备, 可能影响了留学生的学习 积极性。高校攻读学位留学生项目的招生和建设大多是都同时进行的, 只有进一 步加快规范管理和提升教学质量, 才能进入政策依赖度低的良性循坏。

校园环境支持度、师生互动因素对于来华留学生的学习性投入的行为投入、 高阶认知投入、情感投入均具有显著的正向影响。师生互动对学习性投入各维度 的影响均大于校园环境支持度的影响。这一结论呼应了不少对本国学生学习性投 入的研究结果 (Hughes et al., 2008; Lee, 2012; 韩宝平, 2014; 朱红灿, 2014)。

值得一提的是, 生生互动与留学生学习性投入无关这一发现, 与众多研究结 果并不一致 (Kuh, Hu \& Vesper, 2000; Terenzini \& Pascarella, 1991; 朱红灿, 2014)。 我们猜测可能有三个原因。第一, 在华留学生的生生互动 “圈子” 太小, 居住区 域和教学区域集中使得留学生与中国学生的交流受限, 不同生源国留学生之间的 交流也可能因文化风俗或地缘政治受限。第二, 虽然对留学生的学习性投入没有 影响, 生生互动可能会促进他们其他方面的就学体验, 如归属感等。第三, 留学 生的生生互动可能并没有发生在学业学习情境, 正如一位留学生反馈的 “中国学 生之间传来传去的复习资料我们都没有”。学校组织的各类留学生活动可能促进 
了中外学生的文化交流和理解, 但是常规教学活动和学习生活中缺乏同伴互动的 机制。中国学生和教师也须理解来华留学生攻读学位过程中与中国学生面临的同 样甚至更大的学习压力。

\section{基金项目}

本研究获中国高等教育学会外国留学生教育管理分会立项（立项号：高留分会课 题 2016-2017Z005）。

\section{参考文献}

Astin, A. W. (1977). Four critical years. San Francisco, CA, US: Jossey-Bass.

Axelson, R. D., \& Flick, A. (2010). Defining student engagement. Change: The Magazine of Higher Learning, 43(1), 38-43.

Bauer, K. W., \& Liang, Q. (2003). The effect of personality and precollege characteristics on first-year activities and academic performance. Journal of College Student Development, 44(3), 277-290.

Bloom, B. S. (1956). Taxonomy of educational objectives. New York, NY, US: McKay, 20-24.

Brint, S., Cantwell, A. M., \& Hanneman, R. A. (2008). The two cultures of undergraduate academic engagement. Research in Higher Education, 49(5), 383402.

Coates, H. (2005). The value of student engagement for higher education quality assurance. Quality in Higher Education, 11(1), 25-36.

Fazey, D. M., \& Fazey, J. A. (2001). The potential for autonomy in learning: Perceptions of competence, motivation and locus of control in first-year undergraduate students. Studies in Higher Education, 26(3), 345-361.

Finn, J. D. (1993). School engagement and students at risk. Washington, DC, US: National Center for Education Statistics.

Fredricks, J. A., Blumenfeld, P. C., \& Paris, A. H. (2004). School engagement: Potential of the concept, state of the evidence. Review of Educational Research, 74(1), 59109.

Hu, S., \& Kuh, G. D. (2002). Being (dis)engaged in educationally purposeful activities: The influences of student and institutional characteristics. Research in Higher Education, 43(5), 555-575.

Hughes, J. N., Luo, W., Kwok, O. M., \& Loyd, L. K. (2008). Teacher-student support, effortful engagement, and achievement: A 3-year longitudinal study. Journal of Educational Psychology, 100(1), 1-14.

Juvonen, J., Espoinoza, G., \& Knifsend, C. (2012). The role of peer relationships in student academic and extracurricular engagement. In S. L. Christenson, A. L. Reschly, \& C. Wylie (Eds.), Handbook of research on student engagement (pp. 387-401). New York, NY, US: Springer.

Krathwohl, D. R., \& Anderson, L. W. (2009). A taxonomy for learning, teaching, and assessing: A revision of Bloom's taxonomy of educational objectives. Longman.

Kuh, G. D. (2001). Assessing what really matters to student learning inside the national survey of student engagement. Change: The Magazine of Higher Learning, 33(3), $10-17,66$. 
Kuh, G. D., Hu, S., \& Vesper, N. (2000). They shall be known by what they do: An activities-based typology of college students.Journal of College Student Development,41(2), 228-244.

Kuh, G. D., Kinzie, J., Buckley, J. A., Bridges, B. K., \& Hayek, J. C. (2006). What matters to student success: A review of the literature. Commissioned Report for the National Symposium on Postsecondary Student Success: Spearheading a dialog on student success. Retrieved from http://nces.ed.gov/npec/pdf/kuh_team_report.pdf.

Lee, J. S. (2012). The effects of the teacher-student relationship and academic press on student engagement and academic performance. International Journal of Educational Research, 53, 330-340.

Miliszewska, I., \& Horwood, J. (2004). Engagement theory: A framework for supporting cultural differences in transnational education. Paper presented at the 27th HERDSA Annual Conference, Miri, Malaysia.

Newmann, F. M. (1991). Student engagement in academic work: Expanding the perspective on secondary school effectiveness. In J. R. Bliss \& W. A. Firestone (Eds.), Rethinking effective schools: Research and practice (pp. 58-76). Englewood Cliffs, NJ, US: Prentice-Hall.

Pike, G. R. (1999). The effects of residential learning communities and traditional residential living arrangements on educational gains during the first year of college. Journal of College Student Development, 40(3), 269-284.

Pike, G. R. (2000). The influence of fraternity or sorority membership on students' college experiences and cognitive development. Research in Higher Education, 41(1), 117-139.

Pike, G. R., \& Killian, T. S. (2001). Reported gains in student learning: do academic disciplines make a difference? Research in Higher Education, 42(4), 429-454.

Pike, G. R., Schroeder, C. C., \& Berry, T. R. (1997). Enhancing the educational impact of residence halls: the relationship between residential learning communities and first-year college experiences and persistence. Journal of College Student Development, 38(6), 609-621.

Pike, G. R., \& Kuh, G. D. (2005). A typology of student engagement for American colleges and universities. Research in Higher Education, 46(2), 185-209.

Pike, G. R., Smart, J. C., Kuh, G. D., \& Hayek, J. C. (2006). Educational expenditures and student engagement: When does money matter? Research in Higher Education, 47(7), 847-872.

Porter, S. R. (2006). Institutional structures and student engagement. Research in Higher Education, 47(5), 521-558.

Schaufeli, W. B., Martinez, I. M., Pinto, A. M., Salanova, M., \& Bakker, A. B. (2002). Burnout and engagement in university students: A cross-national study. Journal of Cross-cultural Psychology, 33(5), 464-481.

Schaufeli, W. B., Bakker, A. B., \& Salanova, M. (2006). The measurement of work engagement with a short questionnaire: A cross-national study. Educational and Psychological Measurement, 66(4), 701-716.

Schuetz, P. (2008). A theory-driven model of community college student engagement. Community College Journal of Research and Practice, 32, 305-324.

Terenzini, P. T., \& Pascarella, E. T. (1991). Twenty years of research on college students: lessons for future research. Research in Higher Education, 32(1), 83-92. 
Umbach, P. D., \& Wawrzynski, M. R. (2005). Faculty do matter: The role of college faculty in student learning and engagement. Research in Higher education, 46(2), 153-184.

Zepke, N. (2011). Understanding teaching, motivation and external influences in student engagement: How can complexity thinking help?. Research in Post-Compulsory Education, 16(1), 1-13.

岑逾豪. (2014). 本科教学中的高阶学习: 问题、实践和挑战. 复旦教育论坛, 12(2), 47-53.

岑逾豪. (2016). 大学生成长的金字塔模型--基于实证研究的本土学生发展理论. 高 等教育研究, (10), 74-80.

方来坛, 时勘, \& 张风华. (2008). 中文版学习投入量表的信效度研究. 中国临床心理 学杂志, 16(6), 618-620.

韩宝平. (2014). 大学生学习投入影响因素分析. 国家教育行政学院学报, (8), 77-82. 中华人民共和国教育部. 教育统计数据. http://www.moe.gov.cn/s78/A03/moe_560/jytjsj_2017/

靳敏, \& 胡寿平. (2018). 工科专业本科生学习性投入的性别差异分析. 复旦教育论 坛, 16(05), 63-69.

孙超. (2009). 对美国大学生学习产出研究的反思. 高教发展与评估, 25(6), 81-84, 112.

吴明隆. (2010). 问卷统计分析实务: SPSS 操作与应用. 重庆: 重庆大学出版社.

吴素梅, \& 宋彩萍. (2010). 关于高校学生学习性投入状况的调查研究--以上海 w大 学为例. 教育理论与实践, 30(7), 3-6.

张信勇, 市小华, \& 徐光兴. (2008). 大学生的学习投入与人格坚韧性的关系. 心理研 究, 1(6), 72-76.

张轶文, \& 甘怡群. (2005). 中文版 Utrecht 工作投入量表(UWES) 的信效度检验. 中 国临床心理学杂志, 13(3), 268-270, 281.

朱红灿. (2014). 大学生学习投入影响因素的研究--基于学习行为投入、情感投入、 认知投入维度. 高教论坛, (4), 36-40.

\section{作者简介（Author biography）}

供美琼, 上海交通大学高等教育研究院硕士研究生, 中国上海。研究方向: 留学 生学习与发展、学生投入。电子邮箱: joangong@sjtu.edu.cn。

MEIQIONG GONG is a graduate student in the Graduate School of Education at Shanghai Jiao Tong University, China. Her research interests lie in international student learning and development, and student engagement. Email: joangong@sjtu.edu.cn.

岑逾豪 (通讯作者), 上海交通大学高等教育研究院副研究员, 中国上海。研究 方向：学生发展、大学对学生的影响。电子邮箱：ycen@sjtu.edu.cn。

YUHAO CEN (corresponding author) is an Associate Professor in the Graduate School of Education at Shanghai Jiao Tong University, China. Her areas of research interest include student development and college impact on students. Email: ycen@sjtu.edu.cn. 